Finally, this structure is capable of explaining the almost unique behavior of these compounds in readily changing from five-coordinate structures to six-coordinate ones on the addition of suitable ligands ${ }^{1,2,3}$. One of the most fundamental axioms of coordination chemistry, the rule of constancy of coordination number, is violated by these compounds. This has been well established experimentally ${ }^{2,3}$. The ease with which a tetragonal pyramidal structure can change to an octahedral one is obvious and the slight changes observed when these compounds add another ligand are thus readily explainable.
The magnetic criterion is of no use in deciding between the various structures proposed as all these compounds which have been examined have a magnetic moment of one Bohr magneton and at least five structures may be proposed which are consistent with this requirement. The tetragonal pyramidal structure is the only one consistent with the experimental evidence to date as well as being the one which can change to an octahedral structure most readily. The restriction to $\mathrm{d}^{2} \mathrm{sp}^{2}$ or $\mathrm{d}^{2} \mathrm{p}^{3}$ hybridization above is believed to be in line with the requirement that a $d^{2} \mathrm{sp}^{3}$ hybridization should be readily accessible.

\section{Darstellung von 1.3-Bis-diazo-acetessigester und Umwandlung in Diazotetronsäure}

Von F. Weygand, H.-J. Bestmann und H. Fritsche Organisch-chemisches Institut der Techn. Universität Berlin, Berlin-Charlottenburg 2

(Z. Naturforschg. 12 b, 596 [1957] ; eingegangen am 11. Juli 1957)

1.3-Bis-diazoketone sind offenbar noch nicht bekannt. Als ersten Vertreter dieses Verbindungstyps konnten wir den 1.3-Bis-diazo-acetessigester (I) durch Umsetzung von Diazomalonsäure-äthylester-chlorid (nach StAUDinger aus Diazoessigester und Phosgen ${ }^{1}$ ) mit Diazomethan in 70-proz. Ausbeute gewinnen.

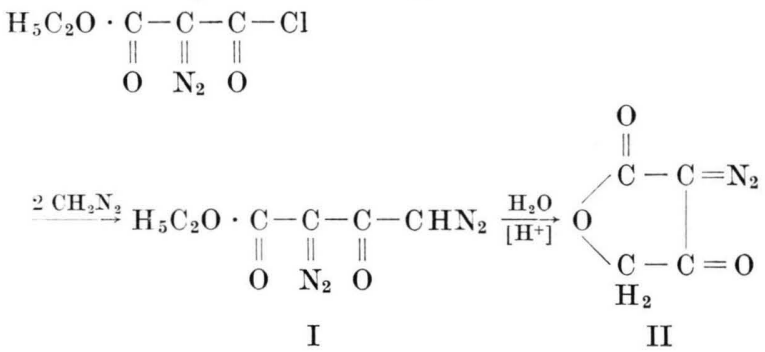

Die Verbindung ist kristallin und gelb gefärbt. Beim Behandeln mit verd. Schwefelsäure erhält man aus ihr

1 H. Staudinger, J. Becker u. H. Hirzel, Ber. dtsch. chem. Ges. 49, 1978 [1916]. die sog. Diazotetronsäure ${ }^{2}$ (II), die auf diesem Wege leicht zugänglich wird.

\section{Beschreibung der Versuche}

1.3-Bis-diazo-acetessigsäure-äthylester: $5 \mathrm{~g}$ Diazo-malonsäure-äthylester-chlorid ${ }^{1}$ in $10 \mathrm{~cm}^{3}$ abs. Äther werden langsam in eine gut gekühlte ätherische Lösung von Diazomethan (aus $16 \mathrm{~g}$ Nitrosomethylharnstoff) eingetropft. Nach 2-stdg. Stehen wird der Äther im Vakuum verdampft. Es hinterbleibt ein Öl, das in der Kälte bald kristallisiert. Nach Umkristallisation aus Äther durch Kühlung auf $-40^{\circ} \mathrm{C}$, feine gelbe Nadeln, Schmp. $53^{\circ}$.

$$
\begin{aligned}
& \mathrm{C}_{6} \mathrm{H}_{6} \mathrm{O}_{3} \mathrm{~N}_{4}(182,1) \text { Ber. C 39,56 H 3,32 N 30,76. } \\
& \text { Gef. C 39,55 H 3,26 N 31,13. }
\end{aligned}
$$

Diazotetronsäure: $5 \mathrm{~g}$ 1.3-Bis-diazo-acetessigsäureäthylester werden in $40 \mathrm{~cm}^{3}$ l-n. $\mathrm{H}_{2} \mathrm{SO}_{4}$ eingetragen, worauf leicht erwärmt wird. Nach beendeter Stickstoffentwicklung wird einige Male mit Methylenchlorid extrahiert, die Methylenchlorid-Lösung mit Natriumsulfat getrocknet und das Lösungsmittel verdampft. Der krist, Rückstand wird aus Alkohol umkristallisiert. Ausbeute $2,65 \mathrm{~g}$ (76,5\% d. Th.), Schmp. und Misch-Schmp. mit einer nach WolfF ${ }^{2}$ dargestellten Probe bei $93^{\circ}$.

$\mathrm{C}_{4} \mathrm{H}_{2} \mathrm{O}_{3} \mathrm{~N}_{2}(126,1)$ Ber. C. 38,10 H 1,60 N 22,22. Gef. C 38,36 H 1,97 N 21,89.

${ }^{2}$ L. WolfF, Liebigs Ann. Chem. 312, 119 [1900]. 